\title{
QCD Functional Integrals for Systems with Nonzero Chemical Potential
}

\author{
Thomas D. Cohen* \\ Department of Physics, University of Maryland \\ College Park, MD 20742 USA \\ E-mail: cohen@physics.umd.edu
}

\begin{abstract}
This paper reviews some recent progress on QCD functional integrals at nonzero chemical potentials. One issue discussed is the use of QCD inequalities for this regime. In particular, the positivity of the integrand of particular Euclidean space functional integrals for two-flavor QCD with degenerate quark masses is used to demonstrate that the free energy per unit volume for QCD with a baryon chemical potential $\mu_{B}$ (and zero isospin chemical potential) is necessarily greater than the free energy with isospin chemical potential $\mu_{I}=\frac{2 \mu_{B}}{N_{c}}$ (and zero baryon chemical potential). This result may be of use in model finite density systems. A corollary to this result is a rigorous $a b$ initio bound on the nucleon mass. The second major issue addressed is the so-called "Silver Blaze" problem: the fact that at zero temperature and chemical potentials less than some critical value the free energy remains as that of the vacuum. This is puzzling in the context of a functional integral since a chemical potential affects the functional determinant of the Dirac operator and any nonzero $\mu$ changes every eigenvalue of the Dirac operator compared to the $\mu=0$ value. The isospin Silver Blaze problem is solved through the study of the spectrum of the operator $\gamma_{0}(\not D+m)$. The status of the baryon Silver Blaze problem is briefly discussed.
\end{abstract}

\section{INTRODUCTION}

The problem of QCD at nonzero density is important both phenomenologically and theoretically. Unfortunately, it is a problem of formidable difficulty.

There is no known analytical way to attack the problem in terms of a convergent systematic expansion except at very high density. In the very high density regime one can use the fact the system is weakly coupled to deduce the form of an interaction kernel between quarks which gives rise to a gap equation yielding color superconductivity [1, 2]. Unfortunately, this regime is only known to be valid at exponentially high densities. Accordingly it is doubtful whether this regime is relevant either in astrophysics or in laboratory experiments.

One might hope to learn about the system via numerical simulations of lattice QCD [3]. Here, too, is a problem. The standard Monte Carlo algorithm relies on a functional with a positive definite measure. Typically finite densities are achieved via a chemical potential and the chemical potential generally yields a functional determinant which is not positive definite and this notorious fermion sign problem spoils the Monte Carlo approach. One way to avoid this is to concentrate on the case of QCD with an isospin chemical potential rather than a baryon one. This has the virtue of having a manifestly real and positive measure in the functional integral [4]. Numerical simulations have been done for this system. [5, 6] Unfortunately, this problem is of little interest phenomenologically since it is relevant to no known physical circumstance either in astrophysics or in a doable terrestrial experiment. There has been recent progress in ways to treat systems with finite baryon chemical potentials. However, these approaches are restricted to the regime of high temperature and low density. While this regime is certainly of some interest and the techniques may be suitable for reaching the QCD critical point (which is certainly of interest) the techniques are not suitable for the interesting regime of relative cold matter which is of real astrophysical interest.

Thus, for much of the regime of interest one is compelled to resort to model building. Clearly it is of interest to see whether there are any new analytically approaches to this class of problems which can provide new insights. Such insights may be of use in furthering theoretical understanding. They also may serve to constrain model building.

This paper reports on two related new developments based on formal properties of the QCD functional integrals at nonzero chemical potentials.

The first is based on the techniques of QCD inequalities [7]. The key insight is that the functional integral for QCD with a finite baryon chemical potential differs from QCD with a finite isospin chemical potential only by a phase. This in turn lets one bound the free energy for QCD with a nonzero baryon chemical potential (and zero isospin chemical potential) by the free energy for QCD with a nonzero isospin chemical potential (with zero isospin chemical potential) [8]. This result is of interest theoretically and may be of importance in constraining model building - since the isospin chemical potential case may be simulated on the lattice, one has a calculable bound which models must

* Work supported in part by the U.S. Department of Energy under grant DE- FG02-93 ER-40762. 
not violate to be consistent with QCD. The method also has a surprising spin-off-it provides a rigorous bound on the mass of the nucleon.

The second development discussed concerns the so-called "Silver Blaze" problem [9]. This problem is named after the famous Arthur Conan Doyle story of that name. In this story Sherlock Holmes used the "curious incident" of a dog doing nothing in the night time as a key clue. In the context of QCD at nonzero chemical potential, the problem arises when one tries to understand the behavior of QCD at zero temperature and small chemical potential via the analysis of functional integrals. We know, of course, that at zero temperature the physical system is unaffected by a chemical potential which is less than some critical value. (For the case of an isospin chemical potential the value is $m_{\pi}$; for the case of a baryon chemical potential it is the energy per nucleon of infinite nuclear matter.) Of course, from the phenomenological perspective this not surprising in the least - until one has a chemical potential equal to the lightest energy per particle number (of the appropriate type) in the spectrum of the system, then at zero temperature the system will remain in its vacuum state. From the point of view of the functional integral, however, this is a curious incident indeed. The chemical potential enters the problem through the functional determinant which is the product of eigenvalues of the Dirac operator. The inclusion of any nonzero chemical potential alters all of the eigenvalues. This leads to the natural expectation that the nonzero chemical potential will affect all functional determinants and thus all functional integrals and thereby all observables. Clearly this does not happen; the question is simply "why not?"

It can legitimately be asked why one should bother trying to understand this problem. This is, quite literally, trying to understand nothing. The significance, however, is that if one wants to ever develop a method based on functional integrals to understand why something happens when the critical chemical potential is exceeded, one has to understand why nothing happens below. The baryon Silver Blaze problem remains unsolved. However, the isospin Silver Blaze problem was solved last year providing new insights into the physics of pion condensation $[9]$.

A word about notation and language. For simplicity of presentation, this paper explicitly discusses the case of QCD with two degenerate flavors $(u$ and $d)$. Everything goes through without change if one includes any number of heavy flavors so long as the chemical potentials associated with these flavors is zero. Thus the phrase "baryon chemical potential" should be taken to mean "the part of the baryon chemical potential associated with the light nonstrange quarks."

In the following section the use of QCD inequalities to constrain QCD at finite baryon chemical potential will be discussed. Following this there will be a short section exploiting the result to rigorously constrain the nucleon mass directly from QCD. The final section is devoted to the Silver Blaze problem. The treatment in sect. ஹ and 1 is largely based on ref. 8] and the discussion borrows heavily from that work, while the work in the final section is principally from ref. [9]. The discussion here, however, is more expansive and considerably more accessible.

\section{QCD INEQUALITIES FOR QCD AT NONZERO CHEMICAL POTENTIAL}

\section{A. A Brief Review of QCD Inequalities}

QCD inequalities are an ideal method to learn some qualitative features of QCD in a rigorous way directly from the theory. Nussinov [10] developed a precursor to the approach with a demonstration that bounds could be placed on various hadronic quantities for a large class of models which were inspired by QCD. The approach itself emerged shortly thereafter with the realization by Weingarten [1] and Witten [12] that analogous bounds could be obtained directly from QCD itself. The key tool to deriving these was the Euclidean space functional integral representations of physical quantities. The method has an undeniable appeal in that one can deduce certain qualitative features of QCD from first principles even while being unable to fully solve the theory. Of course the method is quite limited. One gains qualitative as opposed to quantitative information, and that being only for particular observables. The information gleaned from them is important, however. One role they serve is simply to supplement the understanding obtained from lattice simulations. They also give us an analytic means to understanding some features of QCD which are both observed in the physical world and which can be seen to emerge from lattice studies. As seen here, QCD inequalities can also provide insight and phenomenologically relevant constraints for certain properties of QCD that are not tractable on the lattice using standard Monte Carlo algorithms. The QCD inequality approach is now more than 20 years old and has been reviewed recently [7]. In this subsection a few relevant features will be quickly reviewed so the the results are comprehensible; for more details the reader is directed to see ref. [7].

Before proceeding it is worth noting that the resulting inequalities are not derived with full mathematical rigor. The results cannot strictly be called theorems. However, by the standards of physicists they are quite rigorous; they use only the most vanilla flavored assumptions typically made by physicists. The approach implicitly assumes that the QCD exists as a theory, that it is legitimate to use functional integrals to compute hadronic quantities from the underlying quantum field theory, that the standard Wick rotation to Euclidean space from Minkowski space is 
permitted, and the like. But no additional dynamical assumptions specific to QCD are made.

The key to QCD inequalities is almost trivially simple. One begins in the standard way by relating a physical quantity of interest to a Euclidean functional integral over all possible gauge field configurations. Now suppose a second interesting quantity is found whose functional integral has the following feature: the integrand for the second quantity is greater than or equal to the integrand of the first quantity for every gauge configuration. If one finds such a pair of quantities, it is readily known that the second functional integral is necessarily bigger than the first. Since the two functional integrals are related to physical observables, one immediately derives bounds on the physical quantities.

A bound on the free energy at fixed baryon chemical potential is the focus of this section. In fact, thermodynamically intensive quantities such as free energy densities are not typically studied via QCD inequalities. The method is more commonly applied to the the study of correlation functions which are then used to bound the masses of particles. There is one important example from the past, however, where the approach used intensive quantities: the demonstration by Vafa and Witten [13] where the vacuum energy for QCD with a $\theta$ term has an absolute minimum at $\theta=0$. In fact, this Vafa-Witten paper [13] is the subject of some considerable controversy [14]. However, the controversy concerns the extension of this argument to conclude that parity cannot be spontaneously broken. The underlying demonstration that the vacuum energy has a minimum $\theta=0$ is clearly correct. The Vafa-Witten proof will be discussed next as it provides a template for the bound on the free energy density of QCD at fixed baryon chemical potential.

The derivation by Vafa and Witten is both simple and elegant. The functional integral for the partition function is given by

$$
Z(\theta)=\int D[A] \prod_{i=\text { flavors }} \operatorname{det}\left(\not D+m_{i}\right) e^{-S_{Y M}+i \theta \nu}
$$

where $\operatorname{det}\left(\not D+m_{i}\right)$ is the functional determinant for a particular flavor and is known to be both real and non-negative [1]. The Euclidean space Yang-Mills action is denoted by $S_{Y M}$, and the topological winding number is denoted by $\nu$. Consider what happens when one sets $\theta$ to be nonzero. The only effect of doing this is to include a pure phase factor $e^{i \theta \nu}$ relative to the $\theta=0$ case. Now the rest of the integrand is real and non-negative and the real part of this phase factor is always less than or equal to unity. (We can ignore the imaginary part since we know on physical grounds that it will integrate to zero.) Thus, even without being able to compute the functional integral explicitly one can deduce that the functional integral for the partition function with nonzero $\theta$ is smaller than the partition function with $\theta=0$. But the partition function has a well-known physical meaning: $Z(\theta)=e^{-V E(\theta)}$ where $V$ is the four-dimensional volume and $E(\theta)$ is the vacuum energy as a function of $\theta$. Thus, the bound on the partition function implies that $E(\theta)>E(0)$.

\section{B. A QCD Inequality for Free Energies at Nonzero Chemical Potential}

In this section, a derivation quite analogous to that of Vafa and Witten discussed above is presented. As noted in the introduction, the explicit problem discussed will be for the of two flavor QCD with degenerate quark masses at a nonzero chemical potential. As was also noted in the introduction the generalization to the problem of addition flavors is quite straightforward.

Like the Witten-Vafa case, the starting point is an appropriate free energy density. We begin by considering QCD at fixed temperature and a baryon chemical potential, $G_{B}\left(T, \mu_{B}\right)$. The free energy is related to the grand partition function $Z_{B}\left(T, \mu_{B}\right)$ in the usual way,

$$
G_{B}\left(T, \mu_{B}\right)=-\left(\beta V_{3}\right)^{-1} \log \left(Z_{B}\left(T, \mu_{B}\right)\right),
$$

where $V_{3}$ is the (three-dimensional) volume of the system while $\beta$ is the inverse temperature. The next step is to express this grand partition function as a functional integral. For QCD with two degenerate flavors this is given by

$$
Z_{B}\left(T, \mu_{B}\right)=\int d[A]\left(\operatorname{det}\left(\not D+m-\frac{\mu_{B}}{N_{c}} \gamma_{0}\right)\right)^{2} e^{-S_{Y M}} .
$$

In the preceding equation $N_{c}$ is the number of colors (3 for the physical world), the functional determinant is taken for one quark flavor. Temperature is treated in the standard way via the imposition of boundary conditions: the gluon fields are subject to periodic boundary conditions in time $A(t+\beta)=A(t)$ with $\beta=1 / T$; the fermions in the functional determinant are subject to antiperiodic boundary conditions. Note that while the functional determinant is for a single flavor, it comes in squared reflecting the presence of two flavors in the system. Finally a notational issue should be considered. The chemical potential is for the baryon number (not for the quark number). The fact that the chemical potential is for the baryon number necessitates the factor of $\frac{1}{N_{c}}$ seen in Eq. (3). 
This functional integral cannot be simulated on the lattice via standard Monte Carlo methods. The difficulty is, of course, the fermion sign problem which arises from the functional determinant. The key feature about the inclusion of a nonzero chemical potential is that the functional determinant is, in general, not necessarily real or positive. However, from the perspective of the lattice, what is a major problem from the perspective of QCD inequalities becomes a major opportunity. In particular, it allows one to place an upper bound on the partition function:

$$
Z_{B}\left(T, \mu_{B}\right) \leq \int d[A]\left|\operatorname{det}\left(\not D+m-\frac{\mu_{B}}{N_{c}} \gamma_{0}\right)\right|^{2} e^{-S_{Y M}}
$$

The inequality seen above is clearly quite analogous to the Vafa-Witten case in equality and stems from the identical reason - a phase factor in an otherwise positive definite integrand will always lower the integral relative to an integral with a phase factor of unity.

Of course, as written, inequality (4) is of little interest. While the left-hand side has a clear physical interpretation, the right-hand side at present does not. The insight which enables the approach to be fruitful is that the right-hand side can also be related to a physically meaningful quantity. In particular, it is quite straightforward to see that the right-hand side can easily be related to the free energy density of QCD with an isospin chemical potential [4]. An isospin chemical potential term enters the QCD Lagrangian with the form $\mu_{I} \bar{q} \gamma_{0} \frac{\tau_{3}}{2} q$. The functional integral for the appropriate grand partition function $Z_{I}\left(T, \mu_{I}\right)=\exp \left(-\beta V_{3} G_{I}\left(T, \mu_{I}\right)\right)$ is

$$
Z_{I}\left(T, \mu_{I}\right)=\int d[A] e^{-S_{Y M}} \operatorname{det}\left(\not D+m-\frac{\mu_{I}}{2} \gamma_{0}\right) \operatorname{det}\left(\not D+m+\frac{\mu_{I}}{2} \gamma_{0}\right)
$$

The expression has two functional determinants - one for each flavor - and they have opposite signs in their $\mu_{I}$ terms which encodes the fact that up and down quarks have opposite values for $I_{3}$.

The next steps involve some trivial results of linear algebra:

$$
\gamma_{5}\left(\not D+m+\frac{\mu_{I}}{2} \gamma_{0}\right) \gamma_{5}=\left(-\not D+m-\frac{\mu_{I}}{2} \gamma_{0}\right)=\left(\not D+m-\frac{\mu_{I}}{2} \gamma_{0}\right)^{\dagger}
$$

The final equality is based on the fact that $\not D$ is anti-Hermitian (in Euclidean space) but the unit operator and $\gamma_{0}$ are each Hermitian. The cyclic property of the determinant means that one can express the second functional determinant in Eq. (5) as $\operatorname{det}\left(\not D+m+\frac{\mu_{I}}{2} \gamma_{0}\right)=\operatorname{det}\left(\gamma_{5}\left(\not D+m+\frac{\mu_{I}}{2} \gamma_{0}\right) \gamma_{5}\right)$. This fact along with Eq. (6) gives

$$
\operatorname{det}\left(\not D+m+\frac{\mu_{I}}{2} \gamma_{0}\right)=\left[\operatorname{det}\left(\not D+m-\frac{\mu_{I}}{2} \gamma_{0}\right)\right]^{*}
$$

Using Eq. (7) along with Eq. (5) allows one to deduce that

$$
Z_{I}\left(T, \mu_{I}\right)=\int d[A]\left|\operatorname{det}\left(\not D+m-\frac{\mu_{I}}{2} \gamma_{0}\right)\right|^{2} e^{-S_{Y M}} .
$$

Note that this expression is of the same form as the right-hand side of Eq. (4). Thus we see that inequality (4) together with Eq. (8) yields a useful inequality,

$$
Z_{I}\left(T, \frac{2 \mu_{B}}{N_{c}}\right) \geq Z_{B}\left(T, \mu_{B}\right)
$$

This inequality for the partition functions along with the standard relationship of the free energy to the partition function implies that

$$
G_{B}\left(T, \mu_{B}\right) \geq G_{I}\left(T, \frac{2 \mu_{B}}{N_{c}}\right) .
$$

Inequality (10) is the principal result of this section.

As discussed above, although the results in this paper are derived for two flavor QCD, they can be generalized trivially. It should be immediately clear that the argument goes through without change for QCD with two degenerate light flavors and any number of additional heavy flavors. The only modification is that the chemical potential term must be understood as being the chemical potential associated with the up and down quarks and not the full baryon chemical potential. The reason it goes through is straightforward. The various functional integrals in this more general case include functional determinants for the heavy flavors. However, since, as noted above, the chemical potential only affects the light flavors, these additional functional determinants are real and non-negative. Because the inequalities depend only on the fact that various terms in the functional integrals are real and positive, the presence of these 
extra functional determinants do not alter the preceding inequalities. It is worth noting that this more general case is significant: in nature QCD has two light quarks which are nearly degenerate and have additional heavy flavors.

There is another scenario in which the inequalities hold. Suppose one considers the general case and looks at the full baryon chemical potential (i.e., a chemical potential coupled to all flavors of quarks). Suppose further that one is working in a regime in which the $\bar{s} \gamma_{0} s=\bar{c} \gamma_{0} c=\bar{b} \gamma_{0} b=\bar{t} \gamma_{0} t=0$. In such a regime, the total baryon number in fact comes from up and down quarks so the previous derivation holds. It should be noted that such a regime actually occurs. In particular it happens at zero temperature if one works below the critical chemical potential for strangeness condensation.

It is useful to consider how inequality (10) may prove useful. Recall that standard Monte Carlo methods fail for QCD at finite baryon chemical potential and low temperatures. Moreover, it is generally believed that weak coupling techniques valid at very high densities which lead to nonperturbative phenomena in a manner very similar to conventional BCS theory [1, 2] are thought to work only at extraordinarily high densities. Thus for the foreseeable future all studies of phenomenological significance for relatively cold dense matter will of necessity by based on simplified models [2, 15] rather than QCD. There is nothing wrong with using simplified models; virtually all of the theory of traditional nuclear physics has been made from the perspective of simplified models and not QCD. On the other hand, models need to be constrained in order to be useful. Empirical data is one way to constrain model building. To the extent possible, though, one ought to constrain models directly from QCD. Inequality (10) may prove very useful for this purpose. While one may have to model the left-hand side of the inequality, the right-hand side is amenable to lattice QCD simulations. The reason for this is precisely the same reason the inequality was derivable in the first place: namely, the integrand of the functional integral for $Z_{I}$ is manifestly real and non-negative 4]. Indeed preliminary lattice studies have been done for this quantity both for quenched QCD [5] and for full QCD 6]. These studies have been done on rather small lattices and it is not clear just how reliable they are. However, lattice calculations will undoubtedly improve with time and eventually may provide important constraints on model building through inequality (10).

\section{A BOUND ON THE NUCLEON MASS}

The topic of this section is off of the main line discussed in this article. However, it is worth pursuing since a significant result for the nucleon mass emerges naturally as a corollary to inequality (10). As will be seen, the bound is not very stringent, but it is still of interest because it is a direct result of QCD. Moreover, it provides a solution to a very old problem. Nussinov originally derived a bound in the context of QCD-inspired models: the nucleon mass must be greater than or equal to $\frac{3 m_{\pi}}{2}[10]$. Weingarten, in his seminal paper introducing QCD sum rules, attempted to place a bound on the nucleon as being larger than some multiple of the pion mass 11]. This attempt, unlike that of Nussinov, directly used QCD. However, the attempt failed - the method was only valid for QCD in a world of six or more degenerate light flavors. But this certainly does not correspond to the real world. Weingarten also suggested an alternative approach which did not require six degenerate flavors. However, this method depended on plausible but unproved assumptions about the behavior of the quark propagator in background gauge potentials. Nussinov and Sathiapalan [16] were able to derive a QCD-based bound that $M_{N}>\frac{N_{c} m_{\pi}}{2}$. Their derivation did not rely on $a d$ hoc assumptions about the quark propagator, and it holds for two degenerate flavors. However, the derivation only works in the large $N_{c}$ limit of QCD. Therefore, prior to ref. [8] there were no known rigorous bounds on the nucleon mass from QCD.

One particular remarkable fact about the bound on the nucleon mass obtained in ref. [8] is that it is based on thermodynamic arguments. The usual way masses are bounded in QCD inequalities is via the study of Euclidean space correlation functions.

The derivation begins with the observation that inequality (10) is valid at any temperature and thus applies at $T=0$. The zero temperature system has remarkably simple thermodynamic properties: the system is in a single quantum state (that is to say, thermal fluctuations are completely absent). The quantum state is simply the one that minimizes the $G=H-\mu N$, where $H$ is the Hamiltonian, $G$ is the appropriate free energy and $\mu$ is the appropriate chemical potential (either isospin or baryon); $N=V_{3} \rho$ is the associated particle number. The chemical potential serves to select the quantum state by altering the free energies of the various quantum states according to the particle number. Assuming the spectrum has a gap, an increase in the chemical potential from zero (at zero temperature) will do no nothing until it reaches a critical value where the free energy of a quantum state other than the vacuum drops below the vacuum state. Below this critical value the density must be zero at zero temperature. Note that it is precisely due to the existence of such a critical chemical potential that the Silver Blaze problem arises.

The critical chemical potentials can be defined by the following relations:

$$
G_{B}\left(T=0, \mu_{B}\right)=0 \text { for }\left|\mu_{B}\right|<\mu_{B}^{c},
$$




$$
\begin{aligned}
G_{B}\left(T=0, \mu_{B}\right) & <0 \text { for }\left|\mu_{B}\right|>\mu_{B}^{c}, \\
G_{I}\left(T=0, \mu_{I}\right) & =0 \text { for }\left|\mu_{I}\right|<\mu_{I}^{c}, \\
G_{I}\left(T=0, \mu_{I}\right) & <0 \text { for }\left|\mu_{I}\right|>\mu_{I}^{c} .
\end{aligned}
$$

Inequality (10) along with the relations defining the critical chemical potential (11) imply that

$$
\mu_{B}^{c} \geq \frac{N_{c} \mu_{I}^{c}}{2} .
$$

Now inequality (12) is specified in terms of a critical chemical potential and we want a relation on the nucleon mass. How can we relate the two? The answer is straightforward: $\mu_{B}^{c}$ is bounded from above by the nucleon mass. This can be seen rather trivially from a variational argument. Focus on a quantum state that we know is an eigenstate of the Hamiltonian: a single nucleon at rest. The free energy of this state is known. The energy is $M_{N}$ while the baryon number is unity, thus the free energy is $G_{B}=M_{N}-\mu_{B}$. It is obvious that the free energy of this state is less than zero when $\mu_{B} \geq M_{N}$. This means that there exists a state of lower free energy than the vacuum whenever $\mu_{B} \geq M_{N}$. Of course, it is logically possible that there are states of lower free energy than the vacuum for some value $\mu_{B}$ less than the nucleon mass implying a critical chemical potential of less than $M_{N}$. Indeed, that is what happens in nature. Extrapolations of the masses and densities of finite nuclei (while removing Coulomb effects) to an infinite system [17] lead to the conclusion that in the absence of Coulomb effects, bound infinite nuclear matter forms. Since it is bound the energy per particle is less than that of isolated nucleons. The transition to infinite nuclear matter is first order at zero temperature; just below $\mu_{B}^{c}$ the system has zero energy and zero density, while just above the system has nonzero energy and nonzero density. Thus $\mu_{B}^{c}=M_{N}-B$ where $B$ is the binding energy per nucleon of infinite nuclear matter. For the present purpose the essential observation is that regardless of whether $\mu_{B}^{c}$ is equal to or less than $M_{N}$, it cannot be greater:

$$
\mu_{B}^{c} \leq M_{N}
$$

Inequalities (12) and (13) together yield a bound on the nucleon mass,

$$
M_{N} \geq \frac{N_{c} \mu_{I}^{c}}{2}
$$

Inequality (14) is a principal result of this section. We have succeeded in bounding the nucleon mass from below by another physical observable.

As written, inequality (14) is of limited use. We have no direct way to measure or compute $\mu_{I}^{c}$ without further assumptions, although we do have strong theoretical grounds for believing that $\mu_{I}^{c}=m_{\pi}$. We can, however, turn the inequality around to make a rigorous statement which can be checked. Recall that $\mu_{I}^{c}$ is the energy per unit isospin of the state in QCD with the lowest energy per unit isospin. We can name this state $X$ and denote its mass $m_{X}$ and isospin $I_{X}$ so that $\mu_{I}^{c}=\frac{m_{X}}{I_{X}}$. Inequality (14) can then be written as

$$
M_{N} \geq \frac{N_{c} m_{X}}{2 I_{X}}
$$

where $X$ is some state which exists in QCD. This is a sharp prediction of QCD that can be checked. Taking $X$ to be the pion we see that the inequality is satisfied by more than a factor of 4 .

\section{THE SILVER BLAZE PROBLEM}

\section{A. The Isospin Silver Blaze Problem}

Let us now turn to the Silver Blaze problem. For simplicity of presentation we consider QCD with two degenerate light flavors. In this section we will consider the theory at zero temperature and a nonzero but small chemical potential (either for baryon number or isospin or a combination thereof). For concreteness let us start the discussion for the case of an isospin chemical potential (at zero baryon chemical potential). While this problem is not particularly interesting phenomenologically, it raises many general questions which have analogs in the more general case and has the virtue of being solved [9].

Phenomenologically this system is well understood at low chemical potential [18]. The system remains in the vacuum state with zero energy density and isospin density for all $\left|\mu_{I}\right|$ less than $m_{\pi}$, which serves as the critical point. 
At the critical point there is a second-order phase transition. The state above the transition is a pion condensate. It is very easy to explain this behavior in terms terms of eigenstates of QCD. The $\mu_{I}^{c}$ - the critical value of $\mu_{I}$-is simply the energy per unit isospin for the state of the system with the smallest energy per unit isospin. For this system it is a pion at rest. However, while this interpretation is trivial the connection to the QCD lagrangian remains quite obscure; we have no simple way to obtain the eigenstates starting directly from QCD.

The point of the present study is to try to understand what is going on in terms of a Euclidean space functional integral formulation of the theory. The reason for doing this is twofold. In the first place, Euclidean space functional integrals are a general, powerful, theoretical tool. Secondly, lattice QCD is formulated in terms of them.

The zero temperature limit introduces subtleties. Thus it is simpler to work at finite (but small) temperature at the outset and then consider the limit as $T \rightarrow \infty$ at an appropriate later stage. The key quantity of interest is the free energy. It is given by $G_{I}\left(T, \mu_{I}\right)=E-T S-\mu_{I} I_{3}$ (where $E, T, S$ and $\mu_{I}$ are the energy, temperature, entropy density, isospin and chemical potential, respectively). To help keep things well defined, we work in a finite (but large) box with a volume denoted by $V$. The infinite volume (thermodynamic) limit $\mathrm{V}$ can be taken at the end of the day. In this limit it is natural to express results in terms of intensive quantities such as the energy density, the free energy or isospin density. The free energy is related to the grand partition function in the standard way: $Z_{I}\left(T \mu_{I}\right)=e^{-\beta G\left(T, \mu_{I}\right)}$ (where $\beta=1 / T$ ). As seen in Subsect.【B the grand partition function can be represented as the following functional integral,

$$
Z_{I}\left(T, \mu_{I}\right)=\int d[A]\left|\operatorname{det}\left(\not D+m-\frac{\mu_{I} \gamma_{0}}{2}\right)\right|^{2} e^{-S_{Y M}} .
$$

The essential issue is how the chemical potential influences the free energy. From the functional integral expression it is clear that the chemical potential influences the free energy through the functional determinant of the Dirac operator and only through the functional determinant. We do not know too much about this determinant. But one thing we do know is that the determinant is simply the product of the eigenvalues of the Dirac operator $\operatorname{det}\left(\not D+m-\frac{\mu_{I} \gamma_{0}}{2}\right)=$ $\prod_{j} \lambda_{j}$ where $\left(\not D+m-\frac{\mu_{I} \gamma_{0}}{2}\right) \psi_{j}=\lambda_{j} \psi_{j}$.

This is at the crux of the Silver Blaze problem. If we knew nothing else, it would be natural to assume that for any given gauge field configuration, the eigenspectrum of the Dirac operator with $\mu_{I}=0$ differs from the eigenspectrum with any nonzero $\mu_{I}$. Indeed, it is naturally to expect that every eigenvalue is would be different. The reason for such an expectation is simply the lack of any known reason why the eigenvalues should not depend on $\mu$. Again, in the absence of any other knowledge, one would also naturally assume then that for every gauge configuration, the functional determinant with nonzero $\mu_{I}$ would differ from the functional determinant $\mu_{I}=0$. Since all functional determinants appear to depend on $\mu_{I}$ it is also natural to conclude that $Z_{I}\left(T, \mu_{I}\right)$ must depend on $\mu_{I}$ for any nonzero $\mu_{I}$. Nothing about this expectation seems to depend in a critical way on the temperature; it would seem to hold at $T=0$. Thus one has a natural expectation that at $T=0$ any nonzero chemical potential would alter the free energy. Obviously this expectation is completely wrong. At $T=0$ the free energy is exactly equal to its vacuum value whenever $\left|\mu_{I}\right|<m_{\pi}$. The Silver Blaze problem is about how to understand the "curious incident" of nothing happening to the free energy for the entire regime $\left|\mu_{I}\right|<m_{\pi}$ in the context of a functional integral treatment.

The insight needed for the solution of the isospin Silver Blaze problem is that instead of focusing on the eigenspectrum of the Dirac operator, $\not D+m$, one should instead focus on the eigenspectrum of the $\gamma_{0}$ times the Dirac operator. Why is this of interest? To begin with, note that product rule for determinants implies that

$$
\operatorname{det}\left(\not D+m-\frac{\mu_{I} \gamma_{0}}{2}\right)=\frac{\operatorname{det}\left(\gamma_{0}\left(\not D+m-\frac{\mu_{I} \gamma_{0}}{2}\right)\right)}{\operatorname{det}\left(\gamma_{0}\right)}=\operatorname{det}\left(\gamma_{0}\left(\not D+m-\frac{\mu_{I} \gamma_{0}}{2}\right)\right)
$$

where the last equality exploits the fact that $\operatorname{det}\left(\gamma_{0}\right)=1$. Actually this is a bit of a swindle since the matrices are infinite but it indicates why $\gamma_{0}$ times the Dirac matrix may be of interest. A more legitimate way to express things can be found using some simple linear algebra:

$$
\operatorname{det}\left(\not D+m-\frac{\mu_{I} \gamma_{0}}{2}\right)=\operatorname{det}(\not D+m) \exp \left\{-\frac{1}{2} \int_{0}^{\mu_{I}} \mathrm{~d} \mu_{I}^{\prime} \operatorname{tr} \frac{1}{\gamma_{0}(\not D+m)-\frac{\mu_{I}^{\prime}}{2}}\right\} .
$$

This indicates that a knowledge of the eigenvalues of $\gamma_{0}$ times the Dirac operator at various values of the chemical potential will enable one to find the relevant trace and to do the integral to find the determinant of interest.

The details of how to characterize these eigenstates and compute the determinant are given in detail in Refs. [9, 19]. Most of these technical details are omitted here but a few of the salient results will be noted.

The first important result is that the anti-periodic boundary conditions on the eigenstates in the trace along with the hermiticity properties of the various operators imply that the eigenfunctions of $\gamma_{0}(\not D+m)-\frac{\mu_{I}^{\prime}}{2}$ are arranged 
into families which can be denoted by two indices; an index $j$ representing an "intrinsic" eigenstate, and an index $n$ representing a phase factor indicating which anti-periodic solution one is studying:

$$
\left|\psi_{j n+1}\right\rangle=e^{\frac{i 2 \pi t}{\beta}}\left|\psi_{j n}\right\rangle, \quad \lambda_{j n}=\epsilon_{j}-\frac{\mu_{I}^{\prime}}{2}+i\left(\frac{\phi_{j}}{\beta}+\frac{(2 n+1) \pi}{\beta}\right) .
$$

The eigenvalue of the operator $\lambda_{j n}$ has a real and an imaginary part. To uniquely specify this decomposition a condition on the phase needs to be imposed. Here we take the condition that $-\pi \leq \phi_{j}<\pi$.

The form of Eq. (19) should look familiar. Apart from the phase factor $\phi_{j}$ it is of the same form as for free noninteracting fermions. Of course, in the case of a noninteracting particle, $\epsilon_{j}$ has a simple interpretation: it is the energy of a mode. Thus we will denote $\epsilon_{j}$ (the real part of eigenvalue) as a quasi-energy. These quasi-energies are fundamentally different from energies in some essential ways. In the first place they depend on the background gauge field configuration. Moreover we have no analytic expressions for them.

The trace can be done in two parts: summing over the quasi-energies, and parameterizing the imaginary parts parameterized by the index $n$. The sum over $n$ is the analog of a typical Matsubura sum[20] and can be done explicitly. Straightforward algebra then yields

$$
\begin{aligned}
& \frac{\operatorname{det}\left(\not D+m-\frac{\mu_{I} \gamma_{0}}{2}\right)}{\operatorname{det}(\not D+m)}=\exp \left(-i \sum_{j} \phi_{j} \theta\left(\epsilon_{j}\right) \theta\left(\left|\mu_{I}\right|-2 \epsilon_{j}\right)\right) \\
& \times \exp \left(\frac{\beta}{2} \sum_{j} \theta\left(\epsilon_{j}\right) \theta\left(\left|\mu_{I}\right|-2 \epsilon_{j}\right)\left(\left|\mu_{I}\right|-2 \epsilon_{j}\right)+O\left(e^{-\beta \Lambda}\right)\right)
\end{aligned}
$$

where the fact that we are ultimately interested in the zero temperature limit has been used to obtain $\theta$ functions from hyperbolic tangents which emerge from the Matsubura sum. At this stage we will take the zero temperature limit and neglect the exponentially suppressed terms. Note, however, that this is making the assumption that the quantity of interest, the free energy at nonzero chemical potential, is smooth in the zero temperature limit.

The theta functions in Eq. (20) gives an obvious hint as to how the isospin Silver Blaze problem may be solved. They imply that at zero temperature the functional determinant for any gauge configuration is precisely equal to its $\mu_{I}=0$ value unless $\frac{\left|\mu_{I}\right|}{2}$ is greater than the quasi-energy of the minimum positive quasi-energy mode. Thus, if there is a gap in the quasi-energy spectrum for a given field configuration, then at least for that configuration nothing happens until the gap is reached.

However, this is not sufficient to resolve the Silver Blaze problem by itself. A full resolution requires that a gap in the spectrum exists for the configurations that contribute with nonzero weight to the functional integral at zero temperature. One needs a formal way to specify this and it is naturally given in terms of a spectral density: $\hat{\rho}(\epsilon)$,

$$
\hat{\rho}(\epsilon) \equiv \sum_{j} \delta\left(\epsilon-\epsilon_{j}\right)
$$

where $\epsilon_{j}$ is the $j^{\text {th }}$ quasi-energy (for a given configuration). Using the Matsubura sum and generic properties of the propagator, a very useful expression is obtained for the spectral density []

$$
\hat{\rho}(\epsilon)=\frac{1}{2 \beta} \frac{\partial}{\partial \epsilon} \operatorname{tr}\left[\left(\gamma_{0}(\not D+m)-\epsilon\right)^{-1}+\left((-\not D+m) \gamma_{0}-\epsilon\right)^{-1}\right]+O\left(e^{-\beta \Lambda}\right) .
$$

Next let us introduce a notation to indicate averaging over gauge configurations:

$$
\langle\hat{O}\rangle_{T, \mu_{I}}=\frac{1}{Z_{I}\left(T, \mu_{I}\right)} \int d[A] \hat{O}\left|\operatorname{det}\left(\not D+m-\frac{\mu_{I} \gamma_{0}}{2}\right)\right|^{2} e^{-S_{Y M}} .
$$

We can define the minimum relevant positive quasi-energy, $\epsilon_{\min }:\langle\hat{\rho}(\epsilon)\rangle_{0,0}=0$ if and only if $|\epsilon|<\epsilon_{\min }$.

With these notational preliminaries in place, the isospin Silver Blaze problem is resolved provided that two conditions are satisfied:

$$
\begin{aligned}
& \text { i) } \quad\langle\hat{\rho}(\epsilon)\rangle_{0, \mu_{I}}=\langle\hat{\rho}(\epsilon)\rangle_{0,0} \text { for all } \mu_{I}<2 \epsilon_{\min }, \\
& \text { ii) } \quad \epsilon_{\min }=\frac{m_{\pi}}{2}
\end{aligned}
$$


It is easy to see that these two conditions do indeed resolve the problem. If one uses the relation of the free energy in terms of $Z_{I}$ along with Eqs. (16) and (21) then

$$
\frac{\partial G\left(0, \mu_{I}\right)}{\partial \mu}=2 \int_{0}^{\frac{\mu_{I}}{2}} \mathrm{~d} \epsilon\langle\hat{\rho}(\epsilon)\rangle_{0, \mu_{I}} .
$$

This means that at zero temperature and $\left|\mu_{I}\right|<m_{\pi}$ and if these two conditions are true, the free energy will be independent of $\mu_{I}$ and thus equal to its vacuum value. This in turn means the expectation value of the isospin vanishes.

The validity of these two conditions can be established given one basically innocuous assumption - that there is no first order phase transition for $T=0$ and $\left|\mu_{I}\right|<m_{\pi}$. This assumption is highly plausible from first principles and is known to be true in nature. Condition i) can then be established using straightforward methods which are detailed in ref. [9]. Condition ii) is a bit more interesting. The trick is to study the charged pseudoscalar susceptibility $\chi_{\mathrm{ps}}^{+}=\int \mathrm{d}^{4} x\left\langle J_{-}(x) J_{+}(0)\right\rangle$ (with $\left.J_{+}=\bar{d} \gamma_{5} u\right)$. The key point is that it is expressible as a functional integral; using similar techniques to those discussed above, one obtains

$$
\chi_{\mathrm{ps}}^{+}\left(T, \mu_{I}\right)=\frac{1}{V} \int \mathrm{d} \epsilon \frac{\langle\hat{\rho}(\epsilon)\rangle_{T, \mu_{I}}\left(1+O\left(e^{-\beta \Lambda}\right)\right)}{\left|2 \epsilon-\mu_{I}\right|} .
$$

Thus $\chi_{\mathrm{ps}}^{+}$will diverge when $\frac{\mu_{I}}{2}$ reaches the smallest value of $\epsilon$ for which $\langle\hat{\rho}(\epsilon)\rangle_{0, \mu_{I}}$ is nonzero. Condition i) then implies that this occurs at $\epsilon_{\min }$. Phenomenologically, in the absence of a first order transition, $\chi_{\mathrm{ps}}^{+}$diverges in the infrared when the chemical potential reaches the mass of the lowest excitation with these quantum numbers, namely, $m_{\pi}$. This completes the demonstration.

\section{B. The Baryon Silver Blaze Problem}

The baryon Silver Blaze problem is far more interesting. The problem is how can one use a functional integral formulation to understand how it happens that for $\mu_{B}<M_{N}-B$ (where $B$ is the binding energy for nucleon of nuclear matter ) the system is unchanged from its vacuum. In the first place it is of much greater phenomenological importance than the isospin case. After all, it is at the crux of understanding infinite nuclear matter from QCD. It is also of far more interest theoretically than its isospin cousin. The isospin Silver Blaze problem is resolved in a direct way: all of the functional determinants in the configurations which matter are unchanged from their vacuum values.

Can the baryon Silver Blaze problem be resolved in a similar way? The answer depends on the regime in which one works. First consider a regime in which $0<\mu_{B}<3 m_{\pi} / 2$. In this regime the derivation given for the isospin Silver Blaze problem applies; all of the gauge configurations which contribute have a functional determinant identical to that of the vacuum. We note that there is a paradox associated with the baryon Silver Blaze problem which applies in this regime. From Sect.【 we see that $G_{B}\left(T, \mu_{B}\right) \geq G_{I}\left(T, \frac{2 \mu_{B}}{N_{f}}\right)$ and that this holds at any temperature including $T=0$. The origin of this inequality was simply the phase of the functional determinant. Naively one would expect this phase factor to differ from unity for all gauge configurations since all eigenvalues of the Dirac operator are complex. This in turn leads to an expectation that the inequality should not be saturated, and that $G_{B}\left(T=0, \mu_{B}\right) \geq G_{I}\left(T=0, \frac{2 \mu_{B}}{3}\right)$ for all $\mu_{B}$. But in the present regime this does not happen. Both $G_{B}$ and $G_{I}$ are the vacuum value and, hence, they are equal. Why was the expectation wrong? The derivation in the isospin Silver Blaze problem neatly explains this.

Consider Eq. (20). Note that there are theta functions for the quasi-energies contributing to the phase factor. These are precisely the same theta functions as those for contributions to the magnitude of the functional determinant. Thus, the fact that the configurations which matter to the functional integral have their magnitudes unchanged from their vacuum value in this regime (the resolution of the isospin Silver Blaze problem) also implies that the phases of the functional determinant are unchanged for the relevant configurations. This explains why in this regime $G_{B}=G_{I}$ rather than being less.

Next let us turn to the regime $3 m_{\pi} / 2<\mu_{B}<M_{N}-B$. In this case it is clear that the nature of the solution of the Silver Blaze problem is qualitatively different from the isospin case. To see this let us again return to Sect. [II Recall that the fundamental reason why an inequality was derived in that case was because the integrand for the free energy with a baryon chemical potential differs from the integrand for the free energy with the appropriate isospin chemical potential only due to a phase factor. Now in this kinematic regime we know phenomenologically that $G_{B}\left(T=0, \mu_{B}\right)$ is at its vacuum value while $G_{I}\left(T=0, \frac{2 \mu_{B}}{3}\right)$ is below the vacuum value due to pion condensation. Pion condensation implies that the functional determinants of gauge configurations that contribute are altered from their vacuum value. The only way the baryon chemical potential can leave the free energy unaltered is because of the phase factors. However, this implies a very large conspiracy - the entire effect of the magnitude of the functional determinants 
increasing must be exactly compensated by averaging over the phases. This phenomena is clearly qualitatively quite distinct from the behavior responsible for the isospin Silver Blaze problem.

What is the origin of this conspiracy? At the present time this is unknown. A pessimistic view is that answering this question is tantamount to solving QCD analytically and, hence, is intractable. An optimistic view is that the issue may become clear if one can find a suitable reorganization of the problem. After all, the isospin Silver Blaze problem also looked intractable until it was realized that the key was to express things in term of the eigenvalue of $\gamma_{0}$ times the Dirac operator rather than the Dirac operator itself. Where should we look for hints about how such a reorganization might be accomplished? Although we do not really know, there are some obvious sources for inspiration. One is the region just above $3 m_{\pi} / 2=\mu_{B}$. In this region the relevant configurations have functional determinants slightly larger than at $\mu_{B}=0$ which must be canceled by the phase effects during averaging. Since the functional determinant is small one may be able to derive analytic expressions on the necessary conditions for cancellation which in turn may give a clue as to how things should be organized. The second place to look is in the large $N_{c}$ limit of QCD. A diagrammatic analysis in the large $N_{c}$ limit suggests that effects linking the functional determinant for the up quarks with the functional determinant for the down quarks is suppressed by $1 / N_{c}$. Thus one expects that in the large $N_{c}$ limit the critical chemical potential for the up quark charge and the down quark charge are identical. Clearly this does not happen as it implies the critical baryon chemical potential is just $N_{c} / 2$ times the isospin chemical potential which clearly fails in the chiral limit. Therefore, understanding the breakdown of the large $N_{c}$ approximation for these quantities may well provide a clue.

[1] D. T. Son, Phys. Rev. D 59, 094019 (1999).

[2] Reviews of various aspects of QCD at finite density including color superconductivity can be found in M. Alford, Ann. Rev. Nucl. Part. Sci. 51, 131 (2001); K. Rajagopal and F. Wilczek, in At the Frontier of Particle Physics/Handbook of $Q C D$, ed. M. Shifman (World Scientific, Singapore, 2001) hep-ph/0011333.

[3] The current state of the art of lattice QCD can be found in the Proceedings of 20th International Symposium on Lattice Field Theory (Lattice 2002), Cambridge, MA, USA, 24-29 June 2002, Nucl. Phys. Proc. Suppl. 119 (2003).

[4] M. Alford, A. Kapustin and F. Wilczek, Phys. Rev. D 59, 054502 (1999).

[5] J. B. Kogut and D K. Sinclair, Phys. Rev. D 66, 014508 (2002).

[6] J. B. Kogut and D K. Sinclair, Nucl. Phys. Proc. Suppl. 119, 556 (2003) hep-lat/0209054; Phys. Rev. D 66, 034505 (2002).

[7] A recent review of QCD inequalities may be found in S. Nussinov and M. Lambert, Phys. Rept. 362,193-301 (2002).

[8] T. D. Cohen, Phys. Rev. Lett. 91, 032002 (2003).

[9] T. D. Cohen, Phys. Rev. Lett. 91, 222001 (2003).

[10] S. Nussinov, Phys. Rev. Lett. 51, 2081 (1983).

[11] D. Weingarten, Phys. Rev. Lett. 51, 1830 (1983).

[12] E. Witten, Phys. Rev. Lett. 51, 2351 (1983).

[13] C. Vafa and E. Witten, Phys. Rev. Lett. 53, 535 (1984).

[14] V. Azcoiti and A. Galante, Phys. Rev. Lett. 83, 1518 (1999); T. D. Cohen, Phys. Rev. D 64, 047704 (2001); X. Ji, Phys. Lett. B554, 33 (2003).

[15] Early examples of such model calculations include M. Alford, K. Rajagopal and F. Wilczek, Phys. Lett. B422, 247 (1998); Nucl. Phys. B537, 443 (1999).

[16] S. Nussinov and B. Sathiapalan Nucl. Phys. B168, 285 (1985).

[17] This topic is discussed in any standard nuclear physics textbook. See, for example, P. E. Hodson, E. Gadioli and E. Gadioli Erba, Introductory Nuclear Physics (Clarendon Press, Oxford 1997).

[18] D.T. Son, M.A. Stephanov, Phys. Rev. Lett. 86, 592 (2001); Phys. Atom. Nucl. 64, 834 (2001); Yad. Fiz. 64, 899 (2001).

[19] D. H. Adams, A dimensionally reduced expression for the QCD fermion determinant at finite temperature and chemical potential, hep-th/0401132

[20] The usual treatment is discussed in standard texts on finite temperature field theory or many-body physics. See, for example, Finite-Temperature Field Theory by J. I. Kapusta (Cambridge University Press, Cambridge, 1989) or Quantum Many-Particle Systems by J. W. Negele and H. Orland (Addison-Wesley, Redwood City, 1988). 